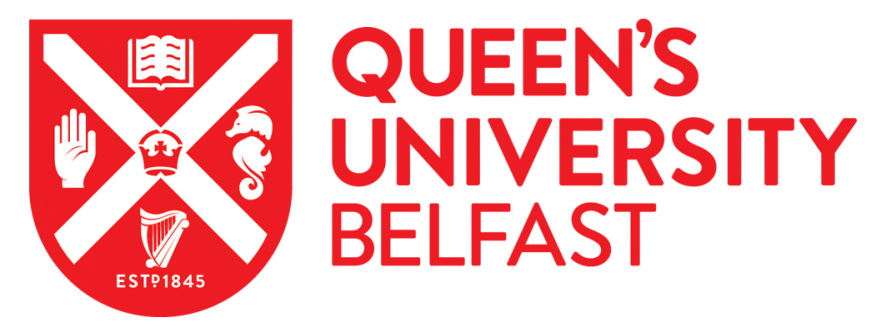

\title{
Linear to Circular Polarization Reflector with Transmission Band
}

Orr, R., Goussetis, G., Fusco, V., \& Saenz, E. (2014). Linear to Circular Polarization Reflector with Transmission Band. 2360-2363. Paper presented at 8th European Conference on Antennas and Propagation, EuCAP 2014, The Hague, Netherlands.

\section{Document Version:}

Peer reviewed version

Queen's University Belfast - Research Portal:

Link to publication record in Queen's University Belfast Research Portal

\section{General rights}

Copyright for the publications made accessible via the Queen's University Belfast Research Portal is retained by the author(s) and / or other copyright owners and it is a condition of accessing these publications that users recognise and abide by the legal requirements associated with these rights.

Take down policy

The Research Portal is Queen's institutional repository that provides access to Queen's research output. Every effort has been made to ensure that content in the Research Portal does not infringe any person's rights, or applicable UK laws. If you discover content in the Research Portal that you believe breaches copyright or violates any law, please contact openaccess@qub.ac.uk. 


\title{
Linear to Circular Polarization Reflector with Transmission Band
}

\author{
R. Orr ${ }^{1}$, G. Goussetis ${ }^{1,2}$, V. Fusco ${ }^{1}$, E. Saenz ${ }^{3}$ \\ ${ }^{1}$ ECIT Institute, Queen's University Belfast, Belfast, United Kingdom, rorr08@qub.ac.uk \\ ${ }^{2}$ School of Engineering and Physical Sciences, Heriot-Watt University, Edinburgh, United Kingdom \\ ${ }^{3}$ European Space Agency, ESA-ESTEC, Noordwijk, The Netherlands
}

\begin{abstract}
A frequency selective surface (FSS) which can be utilized as a diplexer for circular polarization (CP) applications is proposed. The structure consists of two dipole-based FSS placed parallel to each other. The dipoles in one array are rotated by $90^{\circ}$ with respect to those in the other. For an angle of incidence of $45^{\circ}$ at one frequency band the structure allows a $\mathrm{CP}$ signal to be transmitted while at a further band it converts a linearly polarized (LP) signal to CP upon reflection. Full-wave simulation results validated the concept.
\end{abstract}

Index Terms-circular polarization, frequency selective surface, polarization converter.

\section{INTRODUCTION}

Multiband operation can be provided in systems such as a reflector antenna by using a quasi-optical diplexer at the feed arrangement. Frequency Selective Surfaces (FSS) have been used as diplexers in reflector antenna systems together with feeds placed at either side of the FSS [1]-[4]. In such a configuration the FSS is designed to be reflective for one of these feeds acting as a subreflector while for the other it is transparent allowing the feed to be placed at the focal point of the main reflector. Both feeds therefore utilize the same main reflector.

Among the identified diplexer systems to date, all operate in Linear Polarization (LP) [1]-[4]. The advantages of Circular Polarization (CP) operation for communication and sensing systems in terms of simplifying alignment and overcoming Faraday rotation are well known [5]. In this respect, freespace quasi-optical polarization converters are commonly used in satellite communications [6] and imaging systems [7], [8] to transform LP to CP. Many different types of polarizers have been realized including multilayer designs based on meander-line technology [9]-[11] and a single layer design made up of split slot rings [12]. Also, in [13] an anisotropic high impedance surface (HIS) was used as a low profile reflector to provide conversion from LP to CP. None of the available polarization converters provide transmission and reflection bands which is a requirement for operation as a diplexer.

A CP diplexer design should have a transmission and a reflection band. In each of these bands $\mathrm{CP}$ should be conserved, i.e. coincidental TE and TM responses for magnitude and phase would be needed. Multi-channel radiometers are used regularly in earth observation missions to provide information on atmospheric chemistry. Signals which are received at the aperture antenna are separated by FSS in the quasi-optical feed train. Recent requirements for the detection of polarized radiation have led to some FSS designs which provide coincidental spectral responses in the TE and TM planes when excited at $45^{\circ}$ [14]-[16]. Designs such as these would not ensure conservation of $\mathrm{CP}$ as, although the TE and TM magnitudes are coincidental, differences in the phase between TE and TM will affect the axial ratio. Furthermore when using a CP diplexer there is the obvious requirement of $\mathrm{CP}$ feed antennas which complicate the antenna architecture.

In this paper we address the aforementioned limitations proposing a periodic structure that is transparent over one frequency band and fully reflective at another frequency band. The structure is designed to be transparent for TE and TM polarized waves meaning that a $\mathrm{CP}$ signal can be conserved. When illuminated by a linear polarized wave in the reflection band a CP wave is reflected. This polarizing capability in the reflection band reduces the complexity of the antenna architecture as only one CP feed antenna is required. Simulation and design was carried out using CST Microwave Studio (MWS).

\section{OPERATION PRINCIPLE}

The structure consists of two dipole-based FSS parallel to each other placed at a carefully selected distance, h, as displayed in Fig. 1a. The dipoles in one array are rotated by $90^{\circ}$ with respect to those in the other. Excitation is provided by a plane wave at an angle of incidence equal to $45^{\circ}$.

Each FSS is designed to be transparent for both TE and TM polarizations at one frequency (f1). At another frequency (f2) the front FSS fully reflects TE (or TM) polarized waves while is transparent to TM (or TE) polarized waves. Likewise at f2 the back FSS fully reflects TM (or TE) polarized waves. As the FSSs are transparent for both polarizations at the transmission band (around f1) they will not interfere with the signal - the reflector can thus be fed directly with dual CP or LP horn antennas located at the focal point. At the reflection band (around f2), the electrical distance, h, between the FSSs is set 
to be $45^{\circ}$. In this case, when the structure is illuminated by LP at slant $\pm 45^{\circ}$, it will fully reflect the incoming wave in CP. The concept works for $\pm 45^{\circ}$ illumination providing RHCP/LHCP respectively.

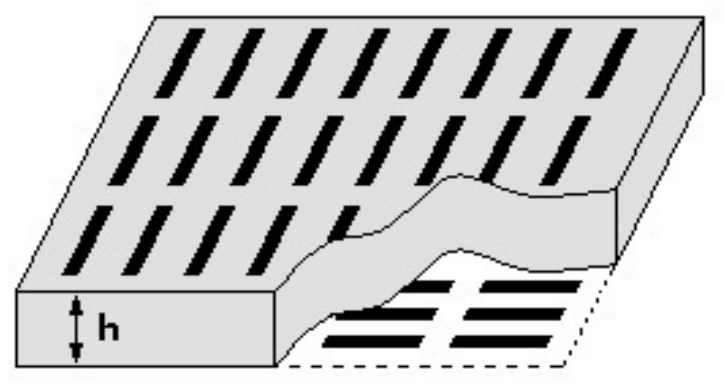

(a)

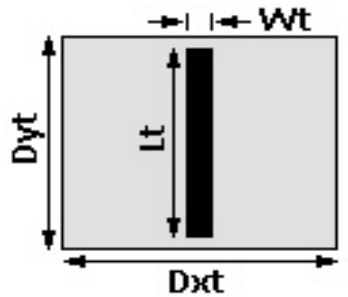

(b)

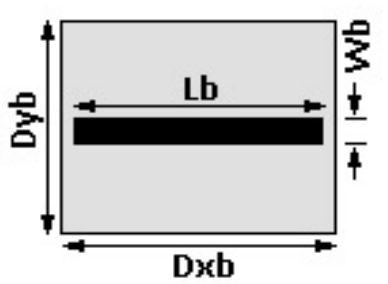

(c)
Fig. 1. Layout of the proposed reflection polarizer with transmission band a) A sample of the structure which consists of two dipole-based FSS separated by a substrate of height, h. b) a unit cell of the top dipole FSS and c) a unit cell of the bottom dipole FSS.

The separation between the FSS, h, required to give CP upon reflection depends on the angle of incidence and the material separating the FSS. For normal incidence and air separating the FSS, h would need to be $\lambda_{0} / 8$. The TE polarized wave would be reflected by the front FSS while the TM would propagate the distance $\mathrm{h}$, twice, reflecting off the back FSS. This provides a phase difference of $90^{\circ}$ between the two polarizations and therefore a CP signal.

In an initial analysis it was assumed that the top FSS was fully reflective for TE polarized waves and the bottom FSS fully reflective for TM polarized waves. An expression was then obtained for the reflection phase difference between the TE and TM components. This expression showed for a given dielectric constant of material between the FSS, separation (h), and angle of incidence at what frequency the required phase difference of $90^{\circ}$ occurred. The design requirements for the surface are: the TE response for the top FSS and TM response for the bottom FSS should be coincident at the reflection and transmission bands, the resonant frequency of each must be at the frequency determined from the expression and the centre frequency of the transmission band must be the same for each FSS.

\section{PERFORMANCE ANALYSIS}

Dipole-based FSS are required for the design of the structure as full transmission is required in the direction orthogonal to the elements. Two FSS unit cell geometries were considered - a single dipole in each unit cell (Fig. 1b and c) or two perturbed dipoles (Fig. 3a and b) in each unit cell. The performance of each geometry type was analyzed to understand which to use in the design. Initially FSS with a single dipole in each unit cell were studied. Fig. $1 \mathrm{~b}$ and $\mathrm{c}$ show the unit cells of the top and bottom FSS with key dimensions indentified. At the resonance frequency of the dipole element full reflection occurs producing a reflection band. Away from the resonance frequency, the reflection magnitude reduces and a transmission band is identified. On this basis the fractional reflection bandwidth was defined as the frequency range over which the reflection magnitude was greater than $-0.3 \mathrm{~dB}$ divided by the frequency of maximum reflection. The fractional separation bandwidth was defined as the difference between the frequencies at which the reflection and transmission magnitudes reduced to $-0.5 \mathrm{~dB}$ divided by the frequency at the mid-point between these two. These were each expressed as percentages.

Modeling and simulation of the FSS were carried out using CST MWS. For the analysis only the TE response of the top FSS was simulated. It was assumed that a similar TM response could be achieved by tuning the element of the bottom FSS. A unit cell of the FSS (Fig. 1b) was constructed and $\mathrm{x}$ - and $\mathrm{y}$-boundaries were set to unit cell implying that the structure was of infinite lateral size. At the z-boundaries floquet ports were set up. In analysis the arrays were freestanding and the conductors made of PEC. The effects of substrate and conductor materials were considered at a later stage. Referring to Fig. $1 \mathrm{~b}$ the initial unit cell dimensions are listed in the caption of Fig. 2. Altering the parameter, $\mathrm{D}_{\mathrm{xt}}$, caused the fractional reflection bandwidth and hence the fractional separation bandwidth to vary. Fig. 2 shows the relationship between fractional reflection and separation bandwidth.

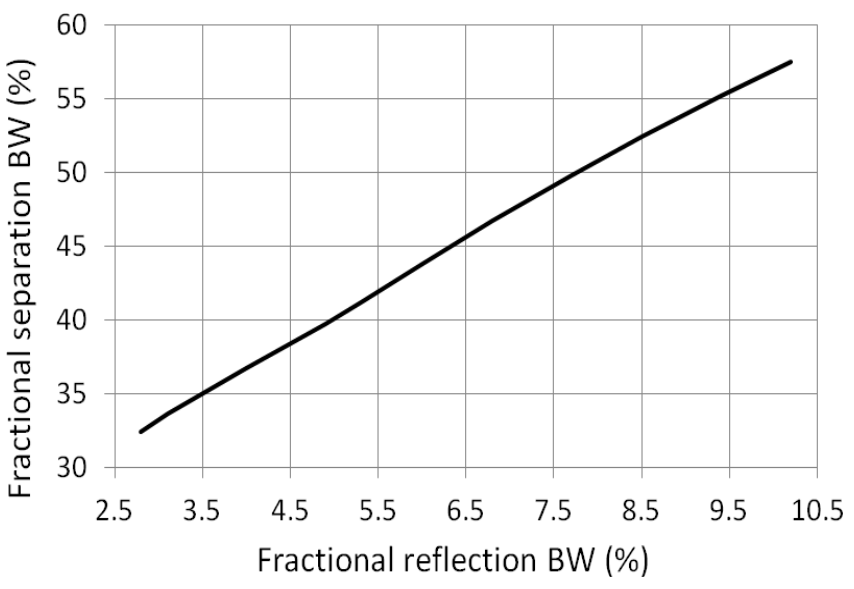

Fig. 2. Relationship between fractional reflection bandwidth and fractional separation bandwidth - for a freestanding dipole FSS, TE polarized wave, angle of incidence $=45^{\circ}$. Initial unit cell dimensions $(\mathrm{mm})$ : Dyt $=\mathrm{Dxt}=5.7$, $\mathrm{Lt}=4.65, \mathrm{Wt}=0.5$.

A linear relationship between fractional reflection and separation bandwidth occurs where an increase in the 
reflection bandwidth results in a greater separation between reflection and transmission bands. A reflection bandwidth of $8 \%$ would result in a separation bandwidth of approximately $51 \%$.

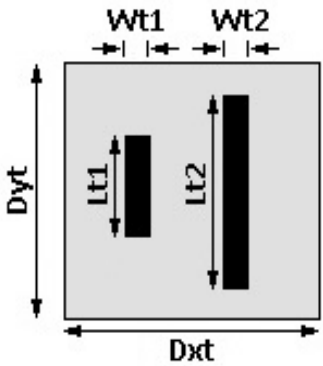

(a)

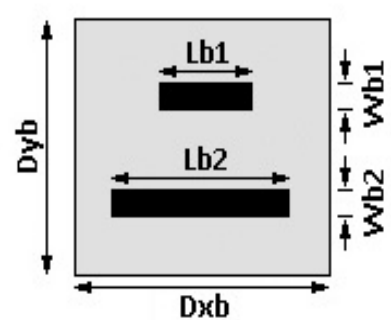

(b)
Fig. 3. A unit cell of a) the top and b) the bottom perturbed dipole FSS.

FSS with two perturbed dipoles in each unit cell were then studied to see if an improved performance could be achieved. Fig. $3 \mathrm{a}$ and $\mathrm{b}$ show the unit cells of the top and bottom FSS. In this analysis fractional transmission bandwidth was defined as the frequency range over which the transmission magnitude was greater than $-0.3 \mathrm{~dB}$ divided by the frequency of maximum transmission and expressed as a percentage. As before a unit cell of the top FSS was created in CST MWS and the TE response was simulated. The initial unit cell dimensions (Fig. 3a) are listed in the caption of Fig. 4. Altering the parameter, $\mathrm{L}_{\mathrm{t} 1}$, caused the fractional separation bandwidth and hence the reflection and transmission bandwidths to vary. Fig. 4 shows the relationship between the fractional separation, reflection and transmission bandwidths.

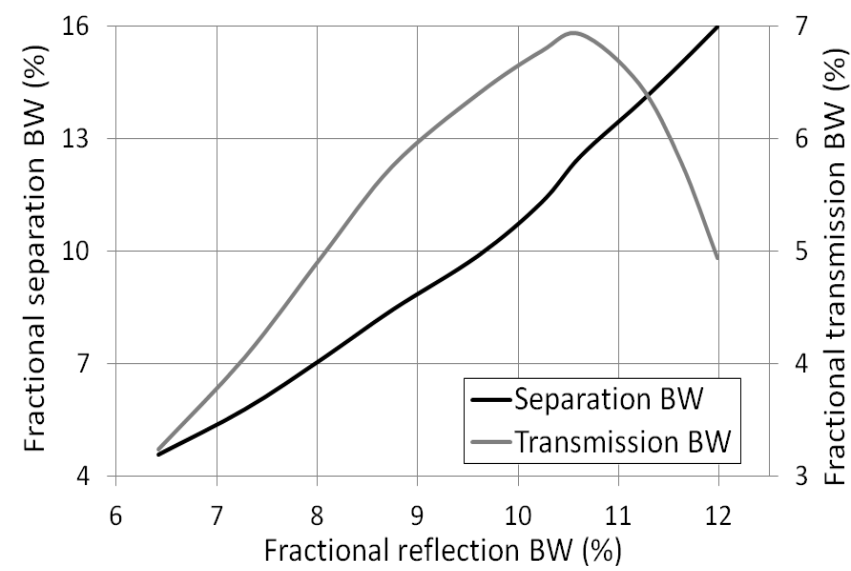

Fig. 4. Relationship between fractional reflection, transmission and separation bandwidths - for a freestanding dipole FSS, TE polarized wave, angle of incidence $=45^{\circ}$. Initial unit cell dimensions $(\mathrm{mm})$ : Dyt $=\mathrm{Dxt}=5.7$, $\mathrm{Lt} 1=3.1, \mathrm{Lt} 2=5.4, \mathrm{Wt} 1=\mathrm{Wt} 2=0.5$

A linear relationship similar to that of the single dipole geometry exists between the fractional separation and reflection bandwidths. The difference when using the perturbed dipole unit cell is that the fractional separation bandwidth can be greatly reduced while maintaining a relatively wide reflection bandwidth. For a single dipole unit cell a reflection bandwidth of $8 \%$ resulted in a separation bandwidth of approximately $51 \%$ whereas when using perturbed dipoles it can be reduced to $7 \%$. As the reflection bandwidth is increased the transmission bandwidth increases to a maximum of approximately $16 \%$ and then reduces.

\section{DESIGN EXAMPLE}

The perturbed dipole geometry was chosen for the design as it allowed the reflection and transmission bands to be brought closer together while maintaining the reflection bandwidth. The perturbed dipole arrays would each be fabricated on TLX-9 substrates (thickness $=130 \mu \mathrm{m}$, permittivity, $\varepsilon_{\mathrm{r}}=2.5$ and loss tangent, $\left.\tan \delta=0.0019\right)$. These would be glued on either side of a $2 \mathrm{~mm}$ thick sheet of Rohacell foam (permittivity, $\varepsilon_{\mathrm{r}}=1.07$ and loss tangent, $\tan \delta=$ 0.0011). Using the derived expression, for the specified substrates and an incident angle of $45^{\circ}$, a $90^{\circ}$ reflection phase difference would occur at approx. $20.5 \mathrm{GHz}$. Therefore the design requirements were: the TE response for the top FSS and the TM response for the bottom FSS should be coincident around the reflection and transmission bands, the resonance frequency of each must be at $20.5 \mathrm{GHz}$ and the transmission band must be centered at the same frequency for each FSS.

CST Microwave Studio (MWS) was used to design each FSS. The unit cell of the top and bottom FSS are displayed with important dimensions identified in Fig. 3a and Fig. $3 b$ respectively. Parametric analyses were carried out to achieve the required results. Referring to Fig. $3 a$ and Fig. $3 b$ the dimensions $(\mathrm{mm})$ were $\mathrm{D}_{\mathrm{yt}}=\mathrm{D}_{\mathrm{xt}}=5.7, \mathrm{~L}_{\mathrm{t} 1}=3.1, \mathrm{~L}_{\mathrm{t} 2}=5.4$, $\mathrm{W}_{\mathrm{t} 1}=\mathrm{W}_{\mathrm{t} 2}=0.5, \mathrm{D}_{\mathrm{yb}}=\mathrm{D}_{\mathrm{xb}}=6.6, \mathrm{~L}_{\mathrm{b} 1}=3.6, \mathrm{~L}_{\mathrm{b} 2}=6.3, \mathrm{~W}_{\mathrm{b} 1}=$ $\mathrm{W}_{\mathrm{b} 2}=0.5$.

Fig. 5 shows the simulated reflection and transmission magnitude for TE and TM polarizations. Fig. 6 displays the reflection and transmission axial ratios. The $3-\mathrm{dB}$ reflection axial ratio $\mathrm{BW}$ is from 19.05 to $22 \mathrm{GHz}$ (approx. 14\%). A global bandwidth was defined as the frequency range over which the reflection or transmission losses were less than 0.5 $\mathrm{dB}$ and the axial ratio was less than $1 \mathrm{~dB}$. For reflection this occurred from 20.1 to $21.1 \mathrm{GHz}(1 \mathrm{GHz}, 5 \%)$. In the case of transmission this occurred from 24.5 to $27 \mathrm{GHz}(2.5 \mathrm{GHz}$, $10 \%)$. The surface has been fabricated, assembled and measured. Measurement results agreed well with those from simulations. The measured results will be presented at the conference. Fig. 7 shows the assembled polarizer structure with the top perturbed dipole FSS visible. The inset shows a zoomed in view of the copper elements on the bottom FSS. 


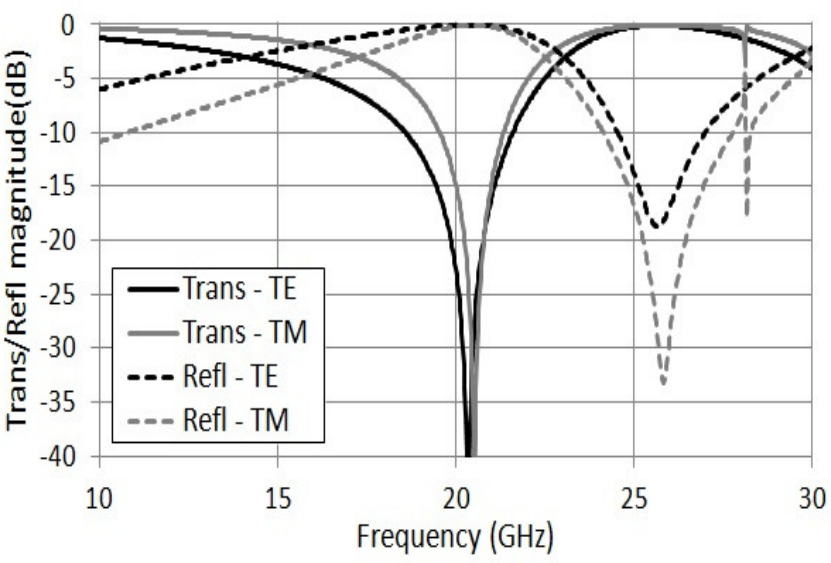

Fig. 5. Simulation results for transmission and reflection magnitude of designed surface - angle of incidence $45^{\circ}-\mathrm{TE}$ and TM polarizations. Unit cell dimensions $(\mathrm{mm}): \mathrm{Dyt}=\mathrm{Dxt}=5.7, \mathrm{Lt} 1=3.1, \mathrm{Lt} 2=5.4, \mathrm{Wt} 1=\mathrm{Wt} 2=$ $0.5, \mathrm{Dyb}=\mathrm{Dxb}=6.6, \mathrm{Lb} 1=3.6, \mathrm{Lb} 2=6.3, \mathrm{~Wb} 1=\mathrm{Wb} 2=0.5$.

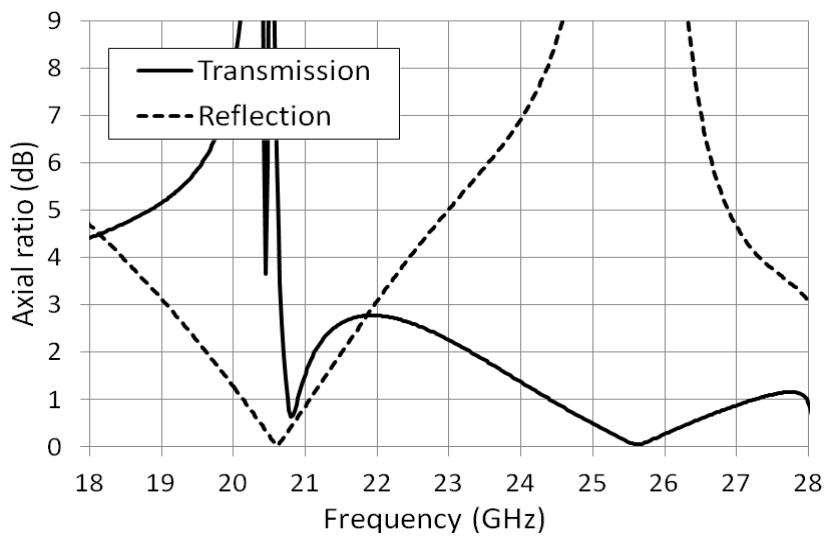

Fig. 6. Simulated reflection and transmission axial ratio - angle of incidence $45^{\circ}$. Unit cell dimensions $(\mathrm{mm})$ : Dyt $=\mathrm{Dxt}=5.7, \mathrm{Lt} 1=3.1, \mathrm{Lt} 2=5.4, \mathrm{Wt} 1=$ $\mathrm{Wt} 2=0.5, \mathrm{Dyb}=\mathrm{Dxb}=6.6, \mathrm{Lb} 1=3.6, \mathrm{Lb} 2=6.3, \mathrm{~Wb} 1=\mathrm{Wb} 2=0.5$.

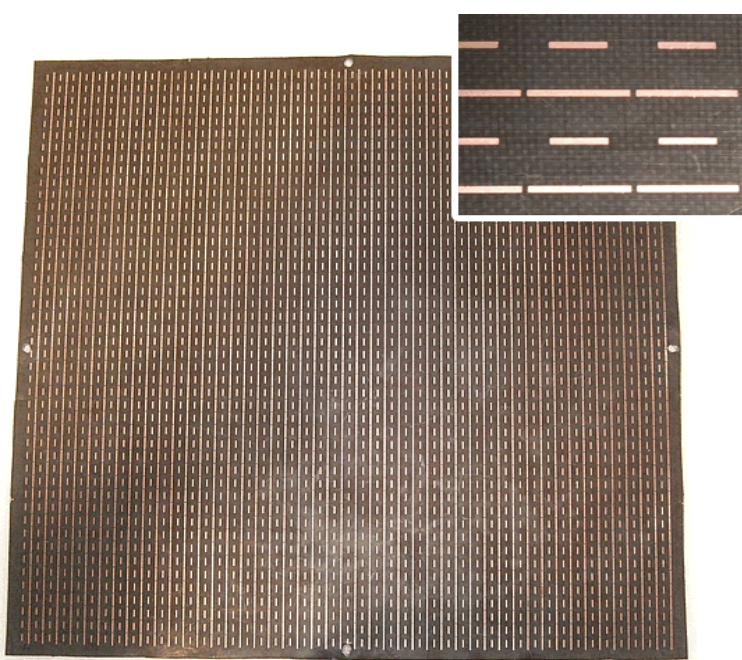

Fig. 7. Prototype of the polarizer with front FSS visible. Inset shows zoomed in view of the back perturbed dipole FSS.

\section{CONCLUSION}

A frequency selective surface which can be used as a diplexer for CP applications has been designed. The structure consists of two perturbed dipole FSS parallel to each other placed at a carefully selected distance. Rohacell foam of the correct thickness separated the FSS. The dipoles in one array are rotated by $90^{\circ}$ with respect to those in the other and the structure is excited by a plane wave at an angle of $45^{\circ}$.

The structure was designed to be transparent for both TE and TM polarizations at one frequency (f1). Around this frequency the FSS would not interfere with the signal and so a reflector could be fed directly with dual CP or LP horn antennas located at the focal point. At a further frequency (f2) the FSS structure was designed to convert LP at slant $\pm 45^{\circ}$ to $\mathrm{CP}$ upon reflection.

Design and simulation of the structure was completed using CST Microwave Studio. A global bandwidth was defined as the frequency range over which the reflection or transmission losses were less than $0.5 \mathrm{~dB}$ and the axial ratio was less than $1 \mathrm{~dB}$. For reflection this was achieved from 20.1 to $21.1 \mathrm{GHz}(5 \%)$. In the case of transmission it was achieved from 24.5 to $27 \mathrm{GHz}(10 \%)$.

\section{REFERENCES}

[1] G. Schennum, "Frequency-Selective Surface for Multiple Frequency Antennas," Microwave Journal, vol. 16, no. 5, pp. 55-57, 1973.

[2] E. Parker and S. Hamdy, "Rings as elements for frequency selective surface," Electron. Lett., vol. 17, no. 17, pp. 613-614, 1981.

[3] R. Cahill and E. Parker, "Concentric ring and jerusalem cross arrays as frequency selective surfaces for a $45^{\circ}$ incidence diplexer," Electron. Lett., vol. 18, no. 8, pp. 313-314, 1982.

[4] C.-C. Huang and N.-W. Chen, "Frequency selective surface for reflector antenna with multiple feeds," 2012 IEEE Antennas and Propagation Society International Symposium (APSURSI),Chicago, Illinois, pp. 1-2, July 2012.

[5] G. Maral and M. Bousquet, Satellite Communications Systems: Systems, Techniques and Technology. Sussex, U.K.: Wiley, 2009, ch. 5, p. 208.

[6] M. Albani et al., "Concepts for Polarising Sheets and "Dual-gridded" Reflectors for Circular Polarisation," ICECom Conference Proceedings, Dubrovnik, Croatia, pp. 1-4, Sep. 2010.

[7] C. Dietlein, A. Luukanen, Z. B. Popovic, and E. Grossman, "A W-band polarization converter and isolator," IEEE Trans. Antennas Propag., vol. 55, no. 6, pp. 1804-1809, Jun. 2007.

[8] K. M. K. H. Leong and W. A. Shiroma, "Waffle-grid polarizer," Electron. Lett., vol. 38, no. 22, pp. 1360-1361, Oct. 2002.

[9] J. Tharp, B. Lail, B. Munk, and G. Boreman, "Design and demonstration of an infrared meanderline phase retarder," IEEE Trans. Antennas Propag., vol. 55, no. 11, pp. 2983-2988, Nov. 2007.

[10] L. Young, L. A. Robinson, and C. A. Hacking, "Meander-line polarizer," IEEE Trans. Antennas Propag., vol. 21, no. 3, pp. 376-378, May 1973.

[11] R.-S. Chu and K. M. Lee, "Analytical model of a multilayered meanderline polarizer plate with normal and oblique plane-wave incidence," IEEE Trans. Antennas Propag., vol. 35, no. 6, pp. 652-661, Jun. 1987.

[12] M. Euler, V. Fusco, R. Cahill, and R. Dickie, " $325 \mathrm{GHz}$ single layer sub-millimeter wave FSS based split ring linear to circular polarization convertor," IEEE Trans. Antennas Propag., vol. 58, no. 7, pp. 24572459, Jul. 2010.

[13] E. Doumanis, G. Goussetis, J. L. Gómez-Tornero, R. Cahill, and V. Fusco, "Anisotropic impedance surfaces for linear to circular polarization conversion," IEEE Trans. Antennas Propag., vol. 60, no. 1, pp. 212-219, Jan. 2012 
[14] R. Dickie, R. Cahill, H. Gamble, V. Fusco, P. Huggard, B. Moyna, M. Oldfield, N. Grant and P. de Maagt, "Polarisation independent bandpass FSS," Electron. Lett., vol. 43, no. 19, pp. 1013-1015, 2007.

[15] R. Dickie, R. Cahill, H. Gamble, V. Fusco, M. Henry, M. Oldfield, P. Huggard, P. Howard, N. Grant, Y. Munro and P. de Maagt, "Submillimeter Wave Frequency Selective Surface With Polarization
Independent Spectral Responses," IEEE Trans. Antennas Propag., vol. 57, no. 7, pp. 1985-1994, 2009.

[16] R. Dickie, R. Cahill, V. Fusco, H. Gamble and N. Mitchell, "THz Frequency Selective Surface Filters for Earth Observation Remote Sensing Instruments," IEEE Trans. Terahertz Sci. Technol., vol. 1, no. 2, pp. 450-461, 2011 . 